\title{
Towards Prediction of Election Outcomes Using Social Media
}

\author{
Vinay K. Jain \\ Department of CSE, Jaypee University of Engineering and Technology, Guna, 473236, India \\ E-mail: vinay2588@gmail.com \\ Shishir Kumar \\ Department of CSE, Jaypee University of Engineering and Technology, Guna, 473236, India \\ E-mail: dr.shishir@yahoo.com
}

Received: 04 March 2017; Accepted: 08 June 2017; Published: 08 December 2017

\begin{abstract}
Exploiting social media data by extracting key information from it is one of the great challenges in data mining and knowledge discovery. Every election campaign has an online presence of voters which uses these social media platform to express their sentiments and opinions towards political parties, leaders and important topics. This paper presents a novel data collection technique for prediction of election outcomes and a topic modeling method for extracting topics. Data collection technique used RSS (Rich Site Summary) feeds of news articles and trending keywords from Twitter simultaneously and constructed an intelligent prediction model based primarily on the volume of tweets and sentiment of users. This paper effort to improve electoral predictions using social media data based dynamic keyword methodology.

Different techniques for electoral prediction based on social media data has been investigated based on existing literature and isolate the factors which improve our methodology. Meaningful inferences such as the popularity of leaders and parties during different intervals, trending issues, and important factors are extracted from the data set. The election outcomes are compared with traditional methods used by survey agencies for exit polls and validation of results showed that social media data can predict with better accuracy. The research has identified that data collection technique and timing play an important role in yielding better accuracy in predicting outcomes and extracting meaningful inferences.
\end{abstract}

Index Terms-Sentiment analysis, Opinion mining, Twitter, Text classification, Feature Selection, Latent Dirichlet allocation(LDA).

\section{INTRODUCTION}

Social media data have been a primary focus in the field of information retrieval (IR) and text mining due to an excessive amount of unstructured data in real time. Every tweet, comment and blog post might reflect their sentiments. These unstructured data provides valuable knowledge which constitutes a big opportunity for creating new services for governments, businesses or individuals [1]. Exploiting these unstructured data created a new field called opinion mining and sentiment analysis.

In the area of opinion mining and sentiment analysis, which deals with the computational calculation of opinion, sentiment, and subjectivity in text, has thus occurred at least in part as a direct response to the surge of interest in new systems that deal directly with opinions More recently, authors developed sentiment analysis methods that can be used across multiple domains like movie and product reviews, election result prediction; disease outbreak, stock market etc [2].This paper focused on election result prediction using the social media data. Indian social media users have rapidly evolved over the past few years to form a complete ecosystem which deals in several areas such as news, politics, health, government policies and finance [3-5].

Nowadays, every election campaign has an online presence and users use these social media to express their sentiments and opinions towards political parties, leaders and important topics during election [6]. Initial studies often presented optimistic results regarding the predictive capacity of Twitter data relative to election results Authors presented optimistic results regarding the predictive capacity of social media towards the election results in different countries [7].The most important factor for better prediction depends on the data or data collection methodology [8]. If the data collected is not much relevant towards the event then the results may be inappropriate [9]. The proposed research focused on getting relevant data from these social media and develops a prediction model which helps in better understanding of election outcomes and meaningful factors. An intelligent data collection technique has been presented using RSS (Rich Site Summary) feeds from top news agencies of India and trending topics from Twitter. Proposed technique is applied to investigate and illustrate meaningful inferences for Delhi Assembly Election 2015[10].Comparison of various machines learning algorithm for classification of data set is carried out. Prediction results are compared with various survey agencies exit polls for validating the results and found 
that results based on social media data can predict with better accuracy. Analysis of social media data also helps in decision making such as finding key political topics, the popularity of the candidate, the impact of debate etc.

The rest of this paper is organized as follows: First, evaluation of various techniques used for prediction the election outcomes in the different geographic region with their data collection methodology. Second, present proposed intelligent techniques for data collection. Third, introduce the features that are relevant and correlated with the election outcomes and propose our prediction model. Fourth, prediction results will be presented with a comparison to exits poll results. Finally, draw conclusions from our experiments and propose our future research.

Table 1. Contribution of different authors for election prediction

\begin{tabular}{|c|c|c|c|c|}
\hline Year & Authors & Country & Election Type & Method(s) \\
\hline 2010 & O'Connor [19] & US & Presidential & $\begin{array}{l}\text { Sentiment Analysis using word } \\
\text { frequencies }\end{array}$ \\
\hline 2010 & Tumasjan[20] & Germany & Federal & Count Tweets/Hashtags \\
\hline 2010 & Hopkins \& King[21] & US, France, Italy & Presidential & $\begin{array}{c}\text { Sentiment classifier based on lexical } \\
\text { induction }\end{array}$ \\
\hline 2010 & Diakopoulos \& Shamma[22] & USA & Presidential & $\begin{array}{c}\text { Demonstrate visuals and metrics of } \\
\text { tweets }\end{array}$ \\
\hline 2011 & Choy[23] & Singapore & Presidential & $\begin{array}{l}\text { Reweighting techniques, Count Tweets } \\
\text { \& Sentiment Analysis }\end{array}$ \\
\hline 2011 & Gayo-Avello[24] & USA & Senate & Count Tweets \& Sentiment Analysis \\
\hline 2011 & Bermingham[25] & Ireland & General & Count Tweets \& Sentiment Analysis \\
\hline 2011 & Metaxas et al. [26] & USA & General & Multiple methods \\
\hline 2011 & Conover et.al. [27] & USA & Congressional midterm & Network clustering algorithm \\
\hline 2011 & Maynard \& Funk[28] & UK & Pre-election & NLP techniques \\
\hline 2012 & Sang [29] & Dutch & Senate & Count Tweets \\
\hline 2012 & Larsson [30] & Sweden & General & Identifying user types \\
\hline 2012 & Choy[31] & USA & Presidential & Count Tweets \\
\hline 2012 & Jungherr [17] & Germany & Federal & Count Hashtags \& Sentiment Analysis \\
\hline 2012 & Skoric et al.[32] & Singapore & General & Volume of tweet \\
\hline 2012 & Tjong \& Bos[33] & Dutch & Senate & Sentiment Analysis \\
\hline 2012 & Marquez et al.[34] & USA. & Presidential & $\begin{array}{l}\text { time series obtained from Twitter } \\
\text { messages }\end{array}$ \\
\hline 2012 & Soler et.al [35] & Spain & Regional and General & Volume of tweets \\
\hline 2012 & Wang et al.[36] & USA & Presidential & Sentiment Analysis \\
\hline 2013 & Contractor [37] & USA & Presidential & NLP techniques \\
\hline 2013 & Cameron[38] & New Zealand & General & Number of Friends/Followers \\
\hline 2013 & Nooralahzadeh[18] & US \& French & Presidential & Sentiment Analysis \\
\hline 2013 & Ceron[39] & Italy \& France & Presidential & Count Tweets \& Sentiment Analysis \\
\hline 2013 & Gaurav[40] & Venezuela & Presidential & Count Tweets \& User \\
\hline 2013 & Wong[41] & USA & Presidential & $\begin{array}{c}\text { Count Tweets/Retweets \& Sentiment } \\
\text { Analysis }\end{array}$ \\
\hline 2013 & Sanders[42] & Netherlands & Parliament & Count Tweets \\
\hline 2013 & Fink[43] & Nigeria & Presidential & Count Tweets \& Sentiment Analysis \\
\hline 2013 & Kim et al.[44] & USA & Presidential election & Iterative topic modeling algorithm \\
\hline 2013 & Bakliwal et al.[45] & Ireland & General & 3-class sentiment classification \\
\hline 2013 & Then \& Ghanem[46] & UK & Members of Parliament & automatic identification \\
\hline 2013 & Vaccari et al.[ 47] & Italian & General election & Volume of tweets \\
\hline 2013 & Vergeer \& Hermans[48] & Netherlands & general & Volume of tweets \\
\hline 2013 & Wong et al.[49] & USA & Presidential & Tweeting and re-tweeting behavior \\
\hline 2014 & Song et al[50] & Korea & Presidential & Text-mining techniques \\
\hline 2014 & Makazhanov[51] & Canada & General & Count of Interactions between Followers \\
\hline 2014 & Ifrim et.al [52] & USA & Presidential & Hierarchical Clustering of Tweets \\
\hline 2014 & Mehndiratta et. al.[53] & India & General & Opinion mining \\
\hline 2014 & Barbera \& Rivero[54] & Spain \&US & $\begin{array}{c}\text { Legislative and } \\
\text { Presidential } \\
\end{array}$ & Volume of tweets \\
\hline 2015 & Vaccari et al.[55] & Italian & General & Volume of tweets \\
\hline 2016 & Tunggawan \& Soelistio[56] & USA & Presidential & Naive Bayesian predictive model \\
\hline 2017 & Jain \& Kumar[5] & India & Legislative Assembly & Emotions based analysis \\
\hline
\end{tabular}




\section{RELATED WORK}

The growth of social media data has interested researchers from various disciplines to uncover the hidden knowledge by applying intelligent data analysis techniques. There are two main types of textual information on the web: facts and opinions. On one hand, facts are assumed to be true and on the other, opinions express subjective information about a certain entity or topic. Some argue that the usage of Twitter and other social media was one of the reasons Obama won the US presidential election in 2008 [11]. Authors present reasons for the increased success of microblogs such as Twitter, noting that their successes come as a result of their unique communication characteristics [12]. A platform such as Xbox gaming is also used for forecasting election [13].Grosse et al.[14] developed a framework which allows mining opinions from Twitter based on incrementally generated queries. Robertson et al., [15] introduced "realized public sphere" in virtual spaces in contrast to political discourse. Hughes and Palen [16] argue in favor of using microblogs as a public information channel used by authorities, for instance in emergency situations. Jungherr et al., [17] argue that methods of prediction using social media analytics are frequently contingent on a somewhat arbitrary experimental variable. Nooralahzadeh et al., [18] used complete keywords by adding the campaign and election hashtags. Most of the authors focused on the volume of the tweets and sentiment analysis based techniques for predicting the election outcomes. Authors used NLP based techniques for an understanding of tweets [5]. Authors also discussed suitable time period for data collection, which is an important feature. In this paper, data collection started before the election dates declaration from the election commission of India.

Table 1 presents a brief review of electoral prediction using social media with a wide variety of techniques used for mining hidden knowledge from the data sets.

\section{Proposed Data Collection Methodology}

In this section, proposed intelligent technique for data collection is presented. The important variable for data collection from social media data are the keywords which help in getting relevant tweets $[57,58]$. Most research for keyword selection used candidate/party names, electionrelated hashtags, and campaign hashtags[18].The period for the conduction of election takes almost 2 to 6 months. During this period lots of topics or events related to the election are discussed in social media, which might be important for predicting the outcomes. Taking this assumption that using more keywords related to the election can collect more relevant data and in turn, increase the prediction accuracy. Proposed data collection methodology presented in Fig. 1, is different from other author's techniques; here we considered those keywords which are trending and dynamic. For getting dynamic keywords three types of data sources are used. Firstly, analysis top newspapers RSS feeds and find out top topics using the Topic modeling technique, LDA (Latent Dirichlet allocation) given by [59]. From these topics, filter out most occurred topics and keywords used in data collection. This methodology gives dynamic keywords which are trending during the election and related to public sentiments. Secondly, use of top trending hashtag taken from Twitter and thirdly, collected from tweets.

\section{A. Keyword Selection}

In this section, identification of dynamic keywords forms three different methods is presented in Fig. 1. These keywords are used for fetching relevant tweets from Twitter. These methods are:

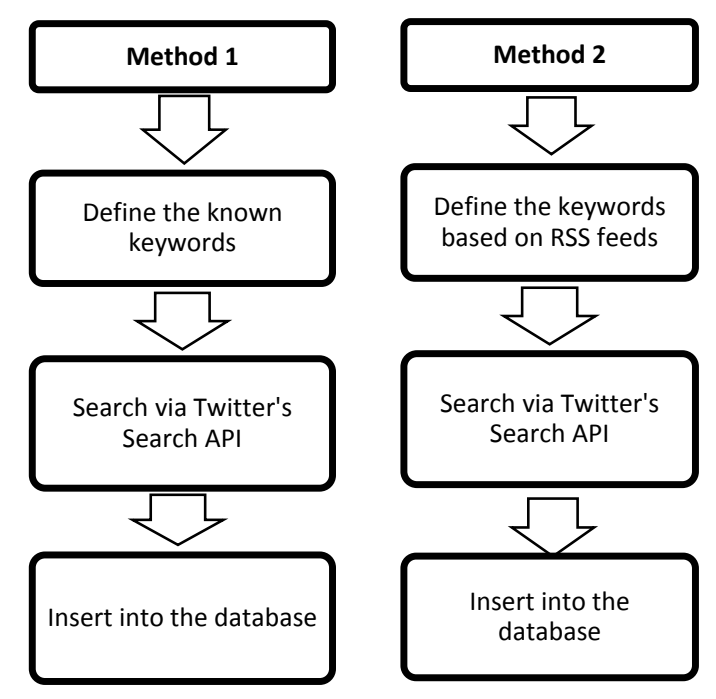

Fig.1. Data collection steps

\section{(i). Keywords from tweets}

Keywords selection is based on TF-IDF (Term Frequency-Inverse Document Frequency) approach which measures the importance of words (or "terms") in a document based on how frequently they appear across multiple documents. Weight is composed of two terms: the first computes the normalized Term Frequency (TF) and the second is to find Inverse Document Frequency (IDF) given in Equation (1) and Equation (2).Define abbreviations and acronyms the first time they are used in the text, even after they have been defined in the abstract. Do not use abbreviations in the title unless they are unavoidable.

$$
\begin{aligned}
& \mathrm{TF}(\mathrm{t})=\frac{\left[\text { Number of times term } \mathrm{t}^{\prime} \text { appears in a document }\right]}{[\text { Total number of terms in the document }]} \\
& \operatorname{IDF}(\mathrm{t})=\log _{\mathrm{e}}\left(\frac{\text { Total number of documents }}{\text { Number of documents with term } \mathrm{t} \text { in it }}\right)
\end{aligned}
$$

\section{(ii). Keywords from RSS feeds}

In this section, RSS (Rich Site Summary) feeds from top news agencies in India have been collected continuously during the period of election and store in the database. Filtering the most important topics are carried out using LDA (Latent Dirichlet allocation) based topic 
modeling approach. After finding the relevant topics, keywords are filtered out based on ranking. According to Blei et al., [59] model, a number of topics $\mathrm{k}$ has to be fixed apriori. For a document $\mathrm{w}=\left(\mathrm{w}_{1}, \mathrm{w}_{2} \ldots, \mathrm{w}_{\mathrm{N}}\right)$ of a corpus D (collection of RSS feeds) containing $\mathrm{N}$ words from a vocabulary consisting of $\mathrm{V}$ different terms, wi $\in$ $\{1, \ldots, \mathrm{V}\}$ for all $\mathrm{i}=1, \ldots, \mathrm{N}$. The steps are illustrated in Fig. 2.

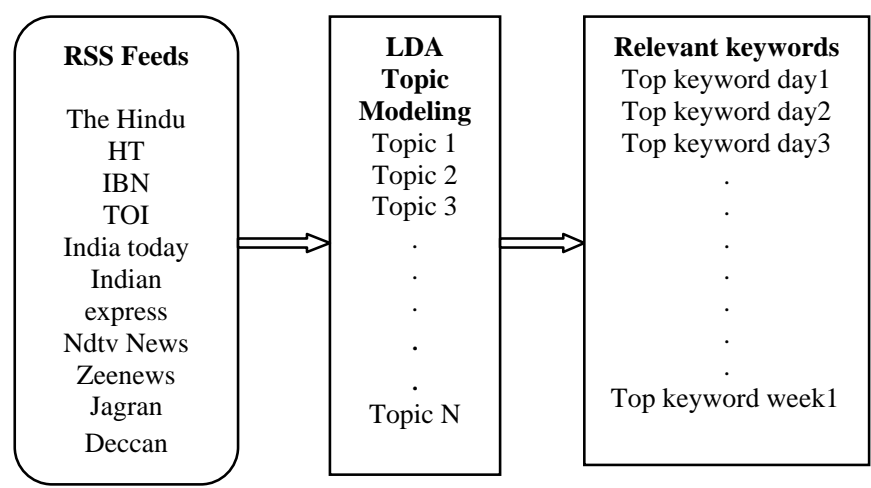

Fig.2. Selection procedure for topics and keywords from RSS feeds

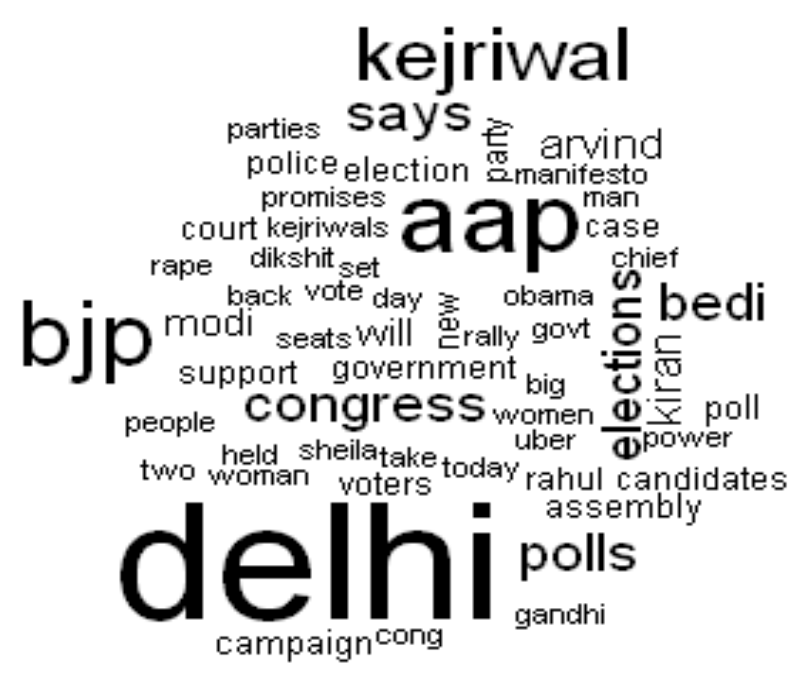

Fig.3. Tag cloud showing most relevant keywords in RSS feeds (02-Jan 15 to $05-$ Feb 15 )

\section{(iii) Trending hashtags from Twitter}

In this section, continuously evaluation and collection of top trending hashtag have been performed during the whole election period. There are various similar keywords are found using these techniques during different intervals and shown in Table 1.

Table 2. Tweets collected during different time intervals with top hashtags

\begin{tabular}{|c|c|l|}
\hline Time Period & $\begin{array}{c}\text { No. of } \\
\text { tweets }\end{array}$ & \multicolumn{1}{|c|}{ Top Keywords } \\
\hline $\begin{array}{c}\text { 20-Nov-2014 to } \\
\text { 20-Dec-2014 }\end{array}$ & 166380 & $\begin{array}{l}\text { \#delhielections, \#delhipolls, } \\
\text { \#Kejriwal, } \\
\text { \#Arvind, \#CrazyKejaria,\#aap, } \\
\text { \#kejriwal, }\end{array}$ \\
\hline $\begin{array}{c}\text { 20-Dec-2014 to } \\
\text { 20-Jan-2014 }\end{array}$ & 212448 & $\begin{array}{l}\text { \#CleanPolitics, } \\
\text { \#vote4aap } \\
\text { \#AAPKiDilli }\end{array}$ \\
\hline $\begin{array}{c}\text { 20-Jan-2015 to } \\
\text { 06-Feb-2015 }\end{array}$ & 312694 & $\begin{array}{l}\text { \#5saalkejriwal, kejriwal4delhi } \\
\text { \#abkibaarbedisarkaar,\#aapkama } \\
\text { nifesto }\end{array}$ \\
\hline
\end{tabular}

\section{B. Data Collection}

After selection of keywords, data collection process is started by retrieving tweets using Twitter. It offers two APIs: Streaming API and REST API for retrieving tweets. The data collection process started before the announcement of the final dates for the election by the Election Commission of India. Continuous data has been fetched from Twitter started from 20th November 2014 to 07th February 2015 using dynamic keywords given in Fig.3. The collected dataset contains 1085721 tweets of which 390425 are re-tweets or duplicates, leaving a total of 703521 tweets. The dataset is divided into three parts based on different time periods.

\section{Data Preprocessing}

Every word contains in a tweet is important in decision making, so pre-processing of these tweets is an important task because these messages are full of slang, misspellings, and words from other languages[60-63]. In order to tackle the problems with the noise in texts, normalization of tweets is performed by applying text preprocessing steps like tokenization, stop words removal, stemming, lemmatization, feature weighting, dimensionality reduction, frequency based methods proposed by Bao et .al[62].

\section{DATA ANALYSIS}

In this section, examination of tweets with different parameters based on the volume of tweets and sentiment analysis of tweets is examined and various meaningful inferences had been extracted. The data analysis technique provides various meaningful inference which helps in the prediction of election outcomes.

\section{A. Volume based analysis}

For detecting the user's voting intention from their tweets towards Delhi Assembly election 2015 has been examined before the declaration of results from Election 
Commission of India. All the major political parties such as Party 1(BJP) Party 2(AAP) and Party 3(Congress) are gearing up for the elections and have formed various strategies for effective campaigning in the city.

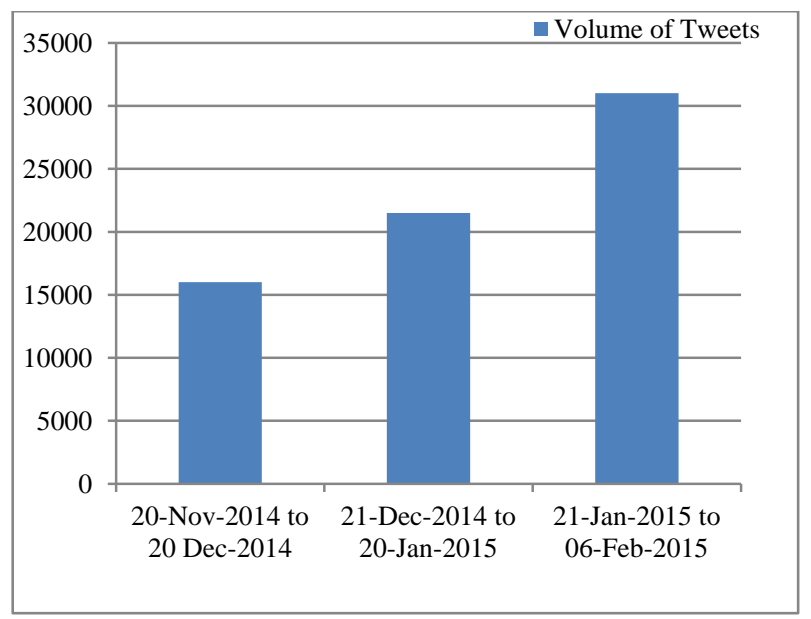

Fig.4. Volume of tweets

The elections are going to be largely between Party 1 and Party 2. The election commission announced the schedule for the elections on 12 January 2015.Date of the poll was $7^{\text {th }}$ February 2015 and counting of the vote is held on $10^{\text {th }}$ February 2015[10].Table 3 and Table 4 presented the total no. of tweets collected from Twitter for corresponding Political parties and their CM candidates.Fig.4 presents total no. of tweets collected.

Table 3. Total tweets count for political party

\begin{tabular}{|c|c|c|c|}
\hline \multicolumn{4}{|c|}{ Total Tweets Counts: $\mathbf{4 2 0 7 0 5}$} \\
\hline Political Party & $\begin{array}{c}\text { Party 1 } \\
\text { (AAP) }\end{array}$ & $\begin{array}{c}\text { Party } 2 \\
\text { (BJP) }\end{array}$ & $\begin{array}{c}\text { Party } 3 \\
\text { (Congress) }\end{array}$ \\
\hline Total & 211500 & 156188 & 53017 \\
\hline
\end{tabular}

Table 4. Total tweets counts for CM candidate

\begin{tabular}{|c|c|c|c|}
\hline \multicolumn{4}{|c|}{ Total Tweets Counts: 216164 } \\
\hline $\begin{array}{c}\text { CM } \\
\text { Candidate }\end{array}$ & $\begin{array}{c}\text { Candidate 1 } \\
\text { (Arvind } \\
\text { Kejriwal) }\end{array}$ & $\begin{array}{c}\text { Candidate 2 } \\
\text { (Kiran Bedi) }\end{array}$ & $\begin{array}{c}\text { Candidate 3 } \\
\text { (Ajay Maken) }\end{array}$ \\
\hline Total & 168183 & 44426 & 3555 \\
\hline
\end{tabular}

During the election time, we find out the popularity of different leaders and know how much user influenced from them. During the election there are many leaders of different party delivered the speech in different rallies related to Delhi election and also talk to media news channels. The political party leader's influence the voters and how much they are popular during this time interval are shown in Fig.5.Gayo-Avello [24] claimed that "conversation" about elections grew as the campaign progressed and elections came nearer. To verify this claim, extracting more keywords and topics related to the election using LDA-based approach in the dataset produce following results presented in Fig. 6.

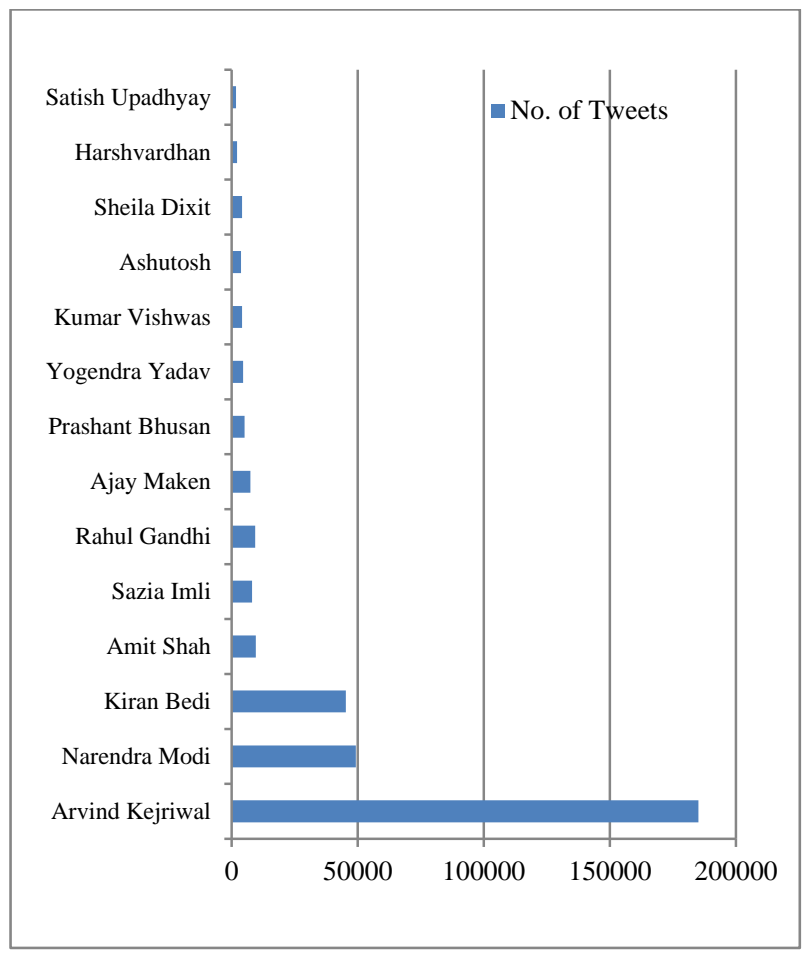

Fig.5. Popularity of leaders during election based on tweets volume

\section{B. Sentiment based analysis}

Proposed framework for sentiment analysis is based on simple opinion and comparison based opinions. Generally, user tweets contain two types of opinions (a) Simple opinion like a vote for Party or vote for Candidate or (b) Comparative opinions like Candidate 1 loyalty vs Candidate 2 or Party 1 vs. Party 2. The system used simple opinion based approach by taking relevant feature for solving the problem of classifying the political sentiment of tweets. 


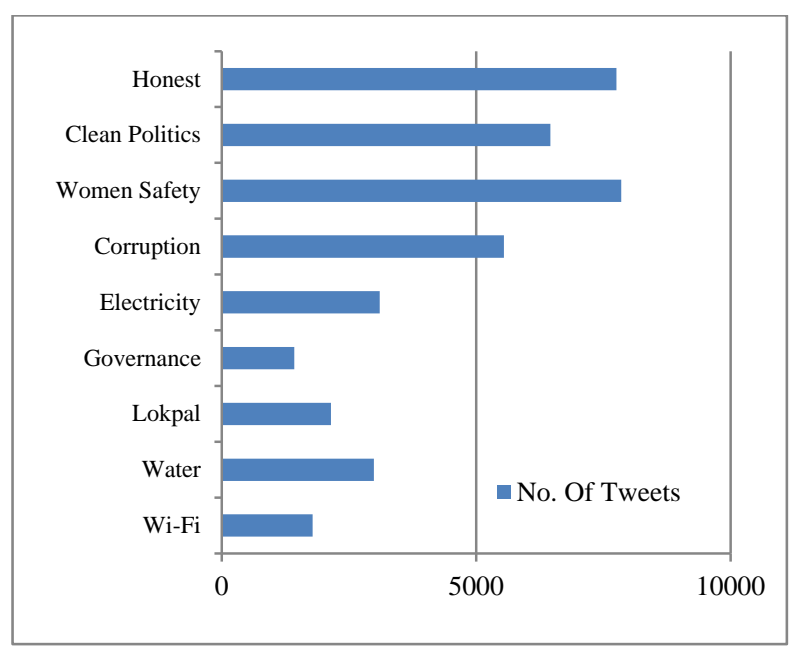

Fig.6. Finding important issues during Delhi election 2015

For building the training and testing set, 1000 tweets during different time intervals are taken. Keywords from the preprocessed dataset are taken as features such as party name, candidate name and their alias. Tweets containing more than one party or candidate name considered as neutral.

For determining the polarity of tweet 4-cross validation of popular machine learning methods are applied such as Support Vector Machine using linear kernel function, Naive Bayes Classifier, Decision tree, Random Forest. Suitable measures are used to assess the efficiency of algorithms, attributes used for classification, training set. Sentiment analysis can help in the analysis the mood for the user.

A comparative performance evaluation of each machine learning methods in terms of the correctly predicted polarity of tweets is examined. The results are explained in terms of precision, recall, accuracy, and Fmeasure and shown in Table 5. The F-measure provides the overall performance of a classifier and is calculated using the following formula:

$$
\mathrm{F}-\text { measure }=\frac{2(\text { Precision })[\text { Recall }]}{\text { Precision }+ \text { Recall }}
$$

Table 5. Performance of the results obtained for each labeled dataset.

\begin{tabular}{|c|c|c|c|c|}
\hline Algorithm & Accuracy & Precision & Recall & F-measure \\
\hline SVM & $79.4 \%$ & 0.798 & 0.785 & 0.792 \\
\hline Naïve Bayes & $69.25 \%$ & 0.685 & 0.714 & 0.699 \\
\hline Random Forest & $77.25 \%$ & 0.786 & 0.749 & 0.767 \\
\hline Decision Tree & $70.80 \%$ & 0.670 & 0.74 & 0.706 \\
\hline
\end{tabular}

From the evaluation, we found that SVM techniques give better results as compared to others machine learning techniques with the accuracy of $79.4 \%$. We get an overall positive sentiment towards Party 1(AAP) and their CM candidate, Candidate 1 .

\section{PREdiction Model}

In this section, a prediction model has been developed to know the user political preference toward a party or candidate in term of percentage of votes. For a given user, $\mathrm{U}=\left\{\mathrm{u}_{1}, \mathrm{u}_{2}, \mathrm{u}_{3} \ldots \ldots \ldots \mathrm{u}_{\mathrm{n}}\right\}$ and a set of parties $\mathrm{P}=\left\{\mathrm{p}_{1}, \mathrm{p}_{2}, \mathrm{p}_{3}\right\}$ or Candidate $\mathrm{C}=\left\{\mathrm{c}_{1}, \mathrm{c}_{2}, \mathrm{c}_{3}\right\}$, examination is carried out as whom $u_{\mathrm{i}}$ has interactions or likely to vote in the upcoming election.

To address the problem, volume based approach has been considered in which a user mentioned the party name, party alias names or the candidate name in their tweets will be considered as the supporter. Using mentioned technique, the percentage of votes for a particular party and the no. of seats it will get has been calculated. Outcomes of the applied technique are presented in comparison to other survey agencies in Table 6 and Fig 7. Results are published before the election results (10-Feb-2015) in Twitter, Facebook and Delhi election forecast (2015) on 03-Feb-2015.

Exit Polls during Delhi election 2015(\% of votes)

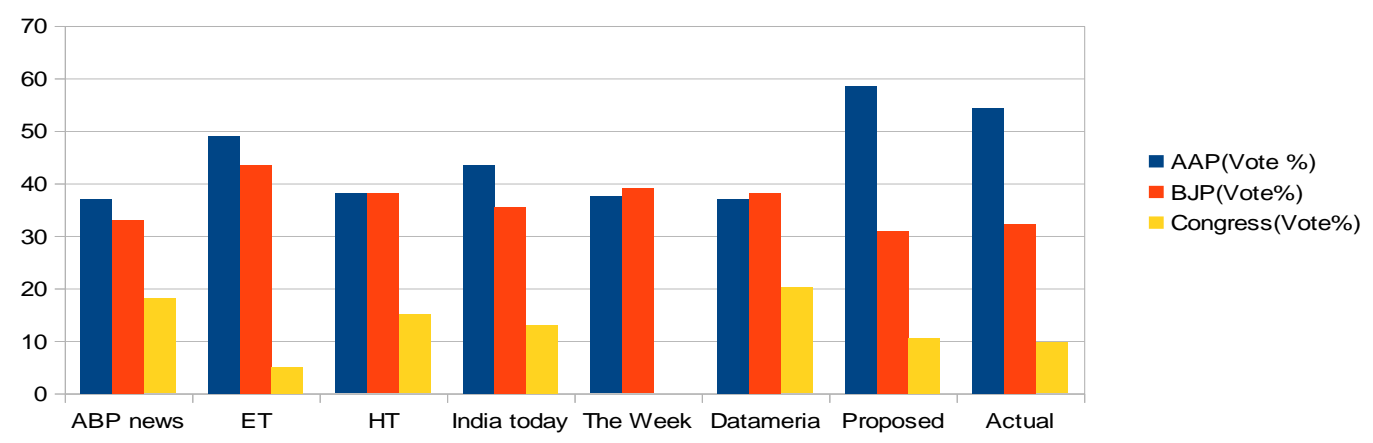

Fig.7.Comparison of exit polls with proposed method [\% of votes] 
Table 6. Comparison of prediction results of survey agencies with proposed method

\begin{tabular}{|c|c|c|c|c|c|}
\hline \multirow{2}{*}{ Date } & \multirow{2}{*}{ Survey agency } & \multirow{2}{*}{ Sample Size } & \multicolumn{3}{|c|}{ Political party } \\
\hline & & & Party 1 & Party 2 & Party 3 \\
\hline 5-7 Nov-14 & ABP News-Nielsen & 6528 & 28 & 36 & 5 \\
\hline 12-Nov-14 & NewsX-CVoter & 2447 & 26 & 37 & 6 \\
\hline 21 Nov-5 Dec -14 & Economic Times-TNS & 7113 & $22-25$ & $43-47$ & $0-3$ \\
\hline 4-8 Dec- 2014 & ABP News-Nielsen & 6409 & 17 & 45 & 7 \\
\hline 18-Dec-14 & India Today-CICERO Wave1 & 4273 & $28 \pm 3$ & $37 \pm 3$ & $4 \pm 1$ \\
\hline 25-Dec-14 & TV24 News India & 8200 & 39 & 23 & 5 \\
\hline 12-Jan-15 & India Today-CICERO Wave 2 & 4459 & $25-30$ & $34-40$ & $3-5$ \\
\hline 12-Jan-15 & India TV-CVoter & 4238 & 29 & 35 & 5 \\
\hline 15-Jan-15 & ABP News-Nielsen & 6414 & 28 & 34 & 8 \\
\hline 11-15 Jan 15 & News Nation & 3195 & $33 \pm 2$ & $31 \pm 2$ & $5 \pm 1$ \\
\hline $10-16$ Jan-15 & Zee News-Taleem & 4200 & 29 & 37 & 4 \\
\hline 18-24 Jan-15 & India TV-CVoter & 1306 & 28 & 37 & 5 \\
\hline 22-24 Jan-15 & The Week-IMRB & 4055 & $29 \pm 2$ & $36 \pm 2$ & $4 \pm 1$ \\
\hline 24-25 Jan-15 & ABP News-Nielsen & 6396 & 35 & 29 & 6 \\
\hline 10-19 Jan-15 & Hindustan Times- $\mathrm{C}$ fore & 7147 & $31-36$ & $31-36$ & $2-7$ \\
\hline 25-31 Jan-15 & India TV-CVoter & 10862 & 31 & 36 & 2 \\
\hline 25-31 Jan-15 & Economic Times-TNS & 3260 & $38 \pm 2$ & $30 \pm 2$ & $2 \pm 1$ \\
\hline 27 Jan- 1 Feb-15 & Hindustan Times- $\mathrm{C}$ fore & 3578 & $36-41$ & $27-32$ & 7 \\
\hline 31 Jan - 1 Feb-15 & AAP [internal] & 3188 & $51 \pm 6$ & $15 \pm 5$ & $4 \pm 2$ \\
\hline 3-Feb-15 & NDTV Poll of Opinion Polls & - & 37 & 29 & 4 \\
\hline 3-Feb-15 & India Today Group-Cicero & 3972 & $42 \pm 4$ & $22 \pm 3$ & $5 \pm 2$ \\
\hline 20 Nov-14 -06 Feb-15 & Proposed Method & 636869 & 42 & 21 & 7 \\
\hline
\end{tabular}

\section{CONCLUSION}

In this paper, a novel intelligent prediction technique for election outcome prediction based on dynamic keywords and topic modeling has been proposed. This technique is applied to electoral prediction during different time intervals using social media data to get the outcomes. This technique is based on the sentiment of voters and volume based technique for prediction. Political Election prediction, results were published on social media, before the declaration by Election Commission of India.It shows how social media sources can help in forecasting election results before the actual results. A brief analysis of important issues regarding elections is also examined. This justifications mentioned in this paper verifies that proposed dynamic keyword based approach can use as next-generation forecasting system for event prediction with better accuracy.

Possibilities of improvement in results are also there, when proposed approach will be applied to the events of those countries where social media is used by most of the citizens for expressing their opinions. Multi-language data preprocessing can improve the prediction accuracy.

\section{REFERENCES}

[1] Vinay K Jain, Shishir Kumar, "Big Data Analytic Using Cloud Computing", in $2^{\text {nd }}$ IEEE International Conference on Advances in Computing and Communication Engineering (ICACCE 2015), Dehradun, pp.667-672, 2015.

[2] B. Pang and L. Lee, "Opinion mining and sentiment analysis", Foundations and Trends in Information
Retrieval, vol.2, pp.1-135, 2008.

[3] Vinay K Jain, Shishir Kumar, "An Effective Approach to Track Levels of Influenza-A (H1N1) Pandemic in India Using Twitter", Procedia Computer Science, Vol.70, pp.801-807, 2015.

[4] Vinay K Jain, Shishir Kumar, “A Novel Approach to Track Public Emotions Related to Epidemics In Multilingual Data", in 2nd International Conference and Youth School Information Technology and Nanotechnology (ITNT 2016),Russia,pp.883-889,May 2016.

[5] Vinay K Jain, Shishir Kumar, "Extraction of Emotions from Multilingual Text Using Intelligent Text Processing and Computational Linguistics" Journal of Computational Science, 2017.(doi: 10.1016/j.jocs.2017.01.010).

[6] M.Anjaria,, R.M.Guddeti, "Influence Factor Based Opinion Mining of Twitter Data Using Supervised Learning”, in Proceeding of Communication Systems and Networks [COMSNETS], 2014 Sixth International Conference ,Banglore, India,pp.1-6,2014.

[7] M.Arias ., A. Arratia, and R. Xuriguera "Forecasting with Twitter Data" ACM Transactions on Intelligent Systems and Technology, Vol. 5, Article no.8,2012.

[8] N.Li,,D. Wu,"Using text mining and sentiment analysis for online forums hotspot detection and forecast", Decision Support Systems, vol.48, pp.354-368,2010.

[9] H.Wang, C.Dogan,A. Kazemzadeh,F. Bar \& S.Narayanan, "A System for Real-time Twitter Sentiment Analysis of 2012 U.S. Presidential Election Cycle", in Proceedings of the 50th Annual Meeting of the Association for Computational Linguistics, Jeju, Republic of Korea, pp.115-120,2012.

[10] Delhi Assembly Election, "Delhi Legislative Assembly election 2015", available at: http://en. wikipedia org /wiki /Delhi_Legislative_Assembly_election,_2015[accessed 15-March-2016]. 
[11] F.Matthew and S.Dutta "Obama's win means future elections must be fought online", available at: http://www.theguardian.com/technology/2008/nov/07/bar ackobama-uselections,2008. [accessed 23-Feb-2015]

[12] F.Wigand,L. Dianne, “ Tweets and retweets: Twitter takes wing in government", Information Polity, vol.16, pp.215$224,2011$.

[13] W.Wang,D. Rothschild ,S.Goel,A. Gelmana“ Forecasting elections with non-representative polls", International Journal of Forecasting, vol.31,pp.980-991,2015.

[14] K.Grosse, M.P. González, C.I. Chesñevar,A.G. Maguitman, A.G., "Integrating argumentation and sentiment analysis for mining opinions from Twitter, AI Communications, Vol.28, pp.387-401,2015.

[15] S.P. Robertson, M. Douglas, B. Maruyama, "Political discourse on social networking sites: Sentiment, ingroup/out-group orientation and rationality", Information Polity, Vol. 18, No. 2,pp. 107-126,2013.

[16] L.A.Hughes and L.Palen "Twitter adoption and use in mass convergence and emergency events", International Journal of Emergency Management, pp. 248-260, 2009.

[17] A. Jungherr,P. Jrgens and H. Schoen, "Why the Pirate Party Won the German Election of 2009", Social Science Computer Review, Vol.30,pp.229-234,2012.

[18] F.A.Nooralahzadeh, "2012 Presidential Elections on Twitter--An Analysis of How the US and French Election were reflected in Tweets", in 19th International Conference on Control Systems and Computer Science [CSCS], Bucharest, pp.240-248,2013.

[19] B. O. Connor, R. Balasubramanyan, B. R.Routledge and N. A.Smith , "From tweets to polls: Linking text sentiment to public opinion time series" in: Proceedings of the International AAAI Conference on Weblogs and Social Media, Washington, DC, May 2010.

[20] A. Tumasjan, T.O. Sprenger, P.Sandner, and I.Welpe, "Predicting Elections with Twitter: What 140 Characters Reveal about Political Sentiment" in International AAAI Conference on Weblogs and Social Media 2010.

[21] D. J.Hopkins and G King, "A Method of Automated Nonparametric Content Analysis for Social Science" American Journal of Political Science, vol.54, pp.229247,2010 .

[22] N. A.Diakopoulos, D. A.Shamma, "Characterizing debate performance via aggregated twitter sentiment" in: $\mathrm{CHI}$ '10 Proceedings of the SIGCHI Conference on Human Factors in Computing Systems ,pp. 1195-1198,2010.

[23] M.Choy , L. F. M.Cheong , M. N.Liak, K. P.Shung, "A sentiment analysis of Singapore Presidential Election 2011 using Twitter data with census correction" Research Collection School Of Information Systems ,2012.

[24] D.Gayo-Avello , "Don't turn social media into another'literarydigest'poll" in: Communications of the $A C M$ vol.54, pp 121-128,2011.

[25] A.Bermingham and A. F. Smeaton , "On using Twitter to monitor political sentiment and predict election results"in International Joint Conference for Natural Language Processing (IJCNLP), Chiang Mai, Thailand,pp.5663,2011.

[26] P. T. A. Metaxas, "How (not) to predict elections: in IEEE third international conference on social computing (SocialCom) ,pp. 165-171,2011.

[27] M. D. Conover, B.Goncalves, J.Ratkiewicz, A.Flammini, and F.Menczer, "Predicting the political alignment of twitter users"in IEEE third international conference on social computing (socialcom),pp. 192-199,2011.

[28] A. D. Maynard and A Funk, "Automatic detection of political opinions in Tweets: in ESWC 2011 Workshops,
Heraklion, Greece,pp 88-99,2011

[29] E. T. Sang, "Predicting the 2011 dutch senate election results with twitter"in Workshop on Semantic Analysis in Social Media, 2012,pp. 53-60.

[30] A. O. Larsson and M Hallvard“Studying political microblogging: Twitter users in the 2010 Swedish election campaign” New Media \& Society, vol.14,729-747,2012.

[31] M. C. Choy, "US Presidential Election 2012 Prediction using Census Corrected Twitter Model" Computers and Society, 2012.

[32] M Skoric , N Poor, P Achananuparp, E P Lim, , and J Jiang, "Tweets and votes: A study of the 2011 singapore general election"in System Science (HICSS), 45th Hawaii International Conference on System Science IEEE,pp. 2583-2591,2012.

[33] E.Tjong, K Sang, J Bos, Predicting the 2011 Dutch Senate Election Results with Twitter" in EACL 2012Workshop on Semantic Analysis in Social Networks, Avignon, France, 2012.

[34] F .B. Marquez, D Gayo-Avello, M Mendoza and B Poblete "Opinion Dynamics of Elections in Twitter "in LA-WEB '12 Proceedings of the 2012 Eighth Latin American Web Congress,pp. 32-39,2012.

[35] J. M.Soler, F Cuartero, M Roblizo, "Twitter as a Tool for Predicting Elections Results" in ASONAM, IEEE/ACM International Conference, 2012, pp.1194 - 1200,2012.

[36] H. Wang, C. Dogan, A. Kazemzadeh ,F. Bar and S. Narayanan, "A System for Real-time Twitter Sentiment Analysis of 2012 U.S. Presidential Election Cycle" in Proceedings of the 50th Annual Meeting of the Association for Computational Linguistics, Jeju, Republic of Korea, pp. 115-120,2012.

[37] D. Contractor, T. A.Faruquie "Understanding Election Candidate Approval Ratings Using Social Media Data, in WWW 2013 Companion, Rio de Janeiro, Brazil,2013.

[38] M. P. Cameron, Can Social Media Predict Election Results? Evidence from New Zealand, Working Paper in Economics 13/08, University of Waikato, 2013.

[39] A. C. Ceron, "Every tweet counts? How sentiment analysis of social media can improve our knowledge of citizens' political preferences with an application to Italy and France" New Media \& Society, vol. 16, 340-358, 2013.

[40] M. S. Gaurav, "Leveraging candidate popularity on Twitter to predict election outcome" in Proceedings of the 7th Workshop on Social Network Mining and Analysis.(ACM) 2013.

[41] F .T. Wong, "Media pundits and the US presidential election: Quantifying political leanings from tweets" in Proceedings of the International Conference on Weblogs and Social Media, 2013.

[42] E. Sanders, "Relating Political Party Mentions on Twitter with Polls and Election Results" in Proceedings of the 13th Dutch-Belgian Workshop on Information Retrieval,pp. 68-71,2013.

[43] C. B. Fink, "Twitter, Public Opinion, and the 2011 Nigerian Presidential Election" in SocialCom, International Conference on Social Computing IEEE,pp.311-320,2013.

[44] H. D. Kim, M. Castellanos, M. Hsu , C. X. Zhai, T. Rietz D. Diermeier, "Mining Causal Topics in Text Data: Iterative Topic Modeling with Time Series Feedback", in CIKM'13, San Francisco, CA, USA,2013.

[45] A. Bakliwal, J. Foster, J. V.Puil, R. O’Brien, L .Tounsi and M. Hughes, "Sentiment Analysis of Political Tweets: Towards an Accurate Classifier" in: LASM, Workshop on Language in Social Media ,Atlanta, Georgia,pp. 49$58,2013$. 
[46] N. A.Thapen, M MGhanem, "Towards Passive Political Opinion Polling using Twitter" in BCS SGAI Workshop on Social Media Analysis , Cambridge, UK.2013.

[47] C.Vaccari, A Valeriani , P.Barberá, R.Bonneau "Social media and political communication: a survey of Twitter users during the 2013 Italian general election" Rivistaitaliana di scienzapolitica, vol.43,pp. 325355,2013

[48] M.Vergeer, L Hermans, "Campaigning on Twitter: Microblogging and Online Social Networking as Campaign Tools in the 2010 General Elections in the Netherlands" Journal of Computer-Mediated Communication, vol.18,pp. 399-419,2013.

[49] F. M. Wong, "Quantifying Political Leaning from Tweets and Retweets" in ICWSM,2013.

[50] M. Song, C M Kim, Y K Jeong, "Analyzing the Political Landscape of 2012 Korean Presidential Election in Twitter",IEEE Intelligent Systems ,vol.1,pp.1541$1672,2013$.

[51] A. R.Makazhanov, "Predicting political preference of Twitter users" Social Network Analysis and Mining, vol.1, pp.1-15,2014.

[52] G.Ifrim, B. Shi, I.Brigadir , "Event Detection in Twitter using Aggressive Filtering and Hierarchical Tweet Clustering",in SNOW 2014 Data Challenge, Seoul, Korea, 2014.

[53] P.Mehndiratta , S Sachdeva, P Sachdeva, Y Sehgal "Elections Again, Twitter May Help!!! A Large Scale Study for Predicting Election Results Using Twitter" in Third International Conference, BDA 2014, New Delhi, India,pp. 133-144,2014.

[54] P.Barbera and G.Rivero, "Understanding the Political Representativeness of Twitter Users", Social Science Computer Review, vol.1, pp.56-67, 2014.

[55] C.Vaccari, A Valeriani, P Barberá, R Bonneau, J T Jost, J Nagler and J A Tucker, "Political Expression and Action on Social Media: Exploring the Relationship Between Lower- and Higher-Threshold Political Activities Among Twitter Users in Italy" Journal of Computer-Mediated Communication,vol.20,pp. 221-239.2015.

[56] Elvyna Tunggawan,Yustinus Eko Soelistio, "And the Winner is ...: Bayesian Twitter-based Prediction on 2016 U.S. Presidential Election” ,International Conference on Computer, Control, Informatics and its Applications.pp.33-37,2016.

[57] B. Narendra, Mr. K. Uday Sai, Mr. G. Rajesh, Mr. K. Hemanth, Mr. M. V. Chaitanya Teja, Mr. K. Deva Kumar "Sentiment Analysis on Movie Reviews: A Comparative Study of Machine Learning Algorithms and Open Source Technologies" I.J. Intelligent Systems and Applications, vol.8, pp.66-70,2016.

[58] Pooja Wadhwa, M.P.S Bhatia, "Discovering Hidden Networks in On-line Social Networks", I.J. Intelligent Systems and Applications, vol.5, pp.44-54, 2014.

[59] David M. Blei, Latent Dirichlet Allocation, Journal of Machine Learning Research, vol.3, pp.993-1022, 2003.

[60] Mayy M. Al-Tahrawi, "Arabic Text Categorization Using Logistic Regression" I.J. Intelligent Systems and Applications, vol.6,pp. 71-78,2015.

[61] Rasim M. Alguliyev,Ramiz M. Aliguliyev and Irada Y. Alakbarova, "Extraction of Hidden Social Networks from Wiki-Environment Involved in Information Conflict", I.J. Intelligent Systems and Applications, vol. 2,pp. 2027,2016.

[62] Y Bao, C Quan,L Wang,F. Ren , "The Role of Preprocessing in Twitter Sentiment Analysis", in ICIC,Taiyuan, China,pp.615-624,2014.
[63] Indrajit Mukherjee,Jasni M Zain,P. K. Mahanti, "An Automated Real-Time System for Opinion Mining using a Hybrid Approach",I.J. Intelligent Systems and Applications, vol.7,p. 55-64,2016.

[64] Ibrahim S. I. Abuhaiba,Hassan M. Dawoud, "Combining Different Approaches to Improve Arabic Text Documents Classification" I.J. Intelligent Systems and Applications, vol.4,pp. 39-52,2017.

\section{Authors' Profiles}

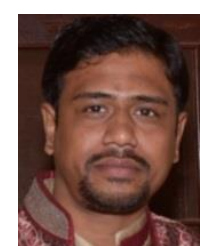

Vinay K. Jain received his B.E. in Computer Science and Engineering in 2009 from Rajiv Gandhi Proudyogiki Vishwavidyala, Bhopal, India and received his M.Tech in Computer Science and Engineering from Jaypee University of Engineering and Technology, Guna, India in 2012. Now, he is pursuing his $\mathrm{Ph} . \mathrm{D}$. degree from Jaypee University of Engineering and Technology, Guna, M.P., India. He has published several papers in peer-reviewed International and Scientific Journals. He is also serving as reviewer for several Science Citation Indexed and Scopus Indexed International Journals.

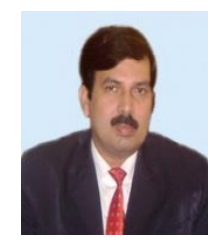

Shishir Kumar in working as Professor the Department of Computer Science and Engineering at Jaypee University of Engineering and Technology, Guna, M.P., India. $\mathrm{He}$ has earned $\mathrm{PhD}$ in Computer Science in 2005 . He has 14 years of teaching and research experience.

How to cite this paper: Vinay K. Jain, Shishir Kumar, "Towards Prediction of Election Outcomes Using Social Media", International Journal of Intelligent Systems and Applications(IJISA), Vol.9, No.12, pp.20-28， 2017. DOI: 10.5815/ijisa.2017.12.03 